\title{
Cost of Revision Total Knee Replacement: A Protocol for Systematic Review and Meta-Analysis
}

\author{
Charles Ebuka Okafor ${ }^{1,2} \oplus$. Son Nghiem ${ }^{1,2} \cdot$ Christopher Vertullo $^{2,3,4} \cdot$ Joshua Byrnes ${ }^{1,2}$
}

Accepted: 9 November 2020 / Published online: 25 November 2020

(c) The Author(s) 2020

\begin{abstract}
Background A major limitation of total knee replacement (TKR), as with other joint replacements, is the risk of revision. Revision TKR is associated with high risk and economic burden to patients, healthcare providers, and societies. It will be worthwhile to assess the economic burden of revision TKR across countries or different study settings. This study aims to review the literature on the cost of revision TKR to assess costs across countries and studies, estimate a pooled cost estimate for homogenous data, and identify major cost components that contribute to the cost burden.

Methods We will conduct a search of the MEDLINE (OvidSp), EMBASE, Web of Science, Cochrane Library, EconLit, and Google Scholar databases to identify relevant studies, and will use an optimally designed search approach to search for relevant studies. EndNote library will be used to manage the searched studies. Selection will be undertaken in two phasesscreening and eligibility. Study selection, data extraction, and assessment of the risk of bias will be performed in duplicate, after which the data will be analysed narratively and a meta-analysis performed for homogenous studies, if possible.

Discussion This protocol provides a proposed stepwise plan for conducting a systematic review and meta-analysis of the cost of revision TKR. Findings from this systematic review will provide information about the cost across settings and identify the major cost drivers of revision TKR, which will, in turn, stimulate efforts to minimize the cost.
\end{abstract}

Systematic Review Registration PROSPERO CRD42020171988.

\section{Background}

One major limitation of total knee replacement (TKR), as with other joint replacements, is the risk of revision. Revision TKR is the reoperation of the TKR procedure due to surgical failure of the primary TKR [1], where some or all of the original prosthesis components are removed and/or replaced, or new components added. The major causes of

Electronic supplementary material The online version of this article (https://doi.org/10.1007/s41669-020-00242-7) contains supplementary material, which is available to authorized users.

Charles Ebuka Okafor

charles.okafor@griffithuni.edu.au

1 Centre for Applied Health Economics, School of Medicine, Griffith University, 170 Kessels Road, Nathan, QLD 4111, Australia

2 Menzies Health Institute, Gold Coast, QLD, Australia

3 Orthopaedic Surgery and Sports Medicine Centre, Gold Coast, QLD, Australia

4 Knee Research Australia, Gold Coast, QLD, Australia revision TKR include infection, instability, aseptic loosening and pain [2]. Approximately $46 \%$ of revision cases occur in the early stage (within 2 years after the primary TKR), while $54 \%$ of revision cases occur 2 years after the primary TKR [3]. The revision rates across countries [2, 4-6], recent epidemiology, and review studies suggest a revision rate of $2.1-9.7 \%$ internationally $[7,8]$.

Revision TKR is associated with higher risk and higher economic burden for patients, healthcare providers (HCPs) and societies compared with primary TKR [9]. The quality of life and survival rate of revision TKR remains much lower than primary TKR [10-12], and with a higher cost [13, 14]. Studies have also shown that revision due to infection costs more than any other cause of revision, such as aseptic loosening [9, 14]. Patients and insurance organizations are faced with the challenge of paying for an unforeseen revision after the primary surgery. Due to the high economic burden, especially in infection-caused revision, some insurance schemes do not cover for the full cost of revision $[9,13]$, which leaves a huge burden on the patients or HCPs. Revision rates can be reduced by optimal patient selection, optimal surgical 
technique, low-risk prosthesis selection and stringent infection prevention methods [15].

Several studies have evaluated the economic burden of revision TKR. In 2010, Oduwole et al. studied the cost of revision TKR to compare the cost difference between aseptic and septic cases of revision and to identify measures to prevent an increase in revision costs [9], while in 2015, Kallala et al. compared revision TKR due to infection and other causes such as pain, aseptic loosening, instability and fracture from an HCP perspective [14]. In 1996, Hebert et al. also compared the cost of revision due to infection and the cost due to primary TKR and aseptic causes of revision from an HCP perspective [13]. The economic burden of revision TKR varies across studies and countries. It will be imperative to review how the economic burden of revision TKR varies across existing studies. It is important to review the studies' characteristics and identify the major cost drivers to inform decisions for future revision TKR in terms of cost minimization. With some insurance schemes paying less than the actual cost of joint revision surgeries, especially in developed countries, cost minimization is important for the sustainability of future procedures [9, 13].

In this study, we propose to review the literature on the reported costs of revision TKR across regions or countries. The problem, intervention, comparator, outcome (PICO) model was used to formulate the research questions. The study intends to address the following questions:

1. What is the cost of revision TKR in different study settings or countries and the pooled cost estimate of homogenous studies?

2. Which cost components contribute significantly to the total cost?

\section{Methods}

\subsection{Protocol and Registration}

The design of this systematic review is in accordance with the recommendations of the Preferred Reporting Items for Systematic Review and Meta-Analysis Protocols (PRISMAP) 2015 statement [16] (details of the PRISMA-P checklist are provided in Online Resource 1). The protocol has been registered in the International Prospective Register of Systematic Reviews (PROSPERO; CRD42020171988).

To aid the development of this protocol, we performed a preliminary assessment of the literature using the PubMed and Google Scholar databases to identify the studies' characteristics and methodologies. This helped us to define our inclusion and exclusion criteria in the search.

\subsection{Eligibility Criteria}

Studies to be included in this review must meet the following criteria.

1. An original economic evaluation of data on revision TKR.

2. Studies must present cost data, either direct cost, indirect cost, or both.

3. Costs from a societal, patient, payer or HCP perspective.

4. Full-text articles and abstracts.

5. Studies on humans that are presented in the English language.

Studies to be excluded in this review include the following.

1. Studies outside the scope of revision TKR.

2. Studies that are not original research, example reviews and commentaries, including opinion papers, conference proceedings and qualitative reports.

3. Cost is not specific for revision TKR or cost of TKR alone without revision cost and cost of readmission without revision. Costs on revision partial knee replacement will also be excluded.

4. Studies with unclear methodology.

\subsection{Information Sources}

The choice of databases to be employed was determined based on the recommendations of expert librarians [17] and after assessment of the health economic core library recommendation by the US National Library of Medicine [18]. We will search the MEDLINE (OvidSp), EMBASE, Web of Science, Cochrane Library, EconLit, and Google Scholar databases to identify relevant studies.

\subsection{Search Strategy}

The search will be performed in November 2020, and we will use an optimally designed search approach to search for relevant studies. Using MEDLINE (OvidSp), we will create concept clusters, with each concept cluster being created by combining a comprehensive set of related search terms. The search terms will be created by combining relevant related Medical Subject Heading (MeSH) terms or combining relevant text words (title, abstract and keywords). Related MeSH terms and text words (search terms) will be combined to form a union (concept cluster). For example, if $A, B$ and $C$ represent related search terms (related MeSH terms and text words), the union is formed as ' $A$ or $B$ or $C$ '. Several relevant concept clusters will be created, which will then 
be combined to form an intersection cluster. For example, if $X, Y$ and $Z$ are concept clusters, they will be combined as ' $X$ and $Y$ and $Z$ '. The results will then be reviewed by looking at the MeSH terms and subheadings, titles and abstract to check if there are terms that could improve our search. For instance, we searched for 'revision knee replacement', which presented results with all headings and subheadings. We then searched for alternative text words, such as 'knee', 'knee revision', 'knee replacement', and 'revision arthroplasty', appearing in the title, abstract, or keywords. For the Cochrane library, we will limit to "bone, joint and muscle trauma, musculoskeletal (MSK), pain palliative and supportive care and injuries in Cochrane group". This search strategy was developed by the research team. Details of the MEDLINE search is shown in Table 1. The MEDLINE search strategy will be adapted for searching in other databases. Auto-alert systems will be set up to provide literature updates while the data extraction and analyses are ongoing. The auto-alert systems will be stopped 2 months after the data extraction.

\subsection{Study Records}

\subsubsection{Data Management}

Results of the search from different databases will be exported into a single EndNote library. Six groups will first be created to contain searches from different databases. EndNote will be used to de-duplicate the studies. After deduplication, we will initiate an auto-search for the full-text of the articles. A union group will be created to contain all the articles. From this union group, we will create different subgroups to represent the exclusion criteria. Excluded studies will be exported to different exclusion folders based on the criteria for exclusion. A study that does not meet the inclusion criteria for multiple reasons will be exported to a relevant exclusion folder in the order of priority: 'scope', 'originality', 'cost analysis/economic evaluation', 'clarity' and 'language'.

\subsection{Selection Process}

The selection process will be independently undertaken by two reviewers, according to the eligibility criteria. A third reviewer will have an overview of the selections made and will resolve any selection disagreement among the first two reviewers. The selection process will be performed in phases. We will first screen titles and abstracts of full-length articles or abstracts of original research articles that involve the cost of revision TKR. Next, we will assess the full-text of potential articles for eligibility, such as clarity, and the perspective of cost used in the study, i.e. whether societal, patient or payer, or HCP. We will also check for the components of the direct and/or indirect cost used. Some relevant but excluded studies (after risk of bias and transparency assessment) in the data synthesis will be listed in a table captioned 'Characteristics of excluded studies'. This will help us to categorize and summarize the studies that meet the inclusion criteria and provide reasons why some relevant studies were excluded. In the eligibility phase, we will use the abstracts of journals where there is no institutional access to full-text and if an application for a subscription will take more than 1 month to complete. See Table 2 for more details on the selection process.

\subsection{Data Collection Process}

We will pilot an electronic data extraction form. Two reviewers will independently extract and manage the data from the included studies. Disagreement with the extraction results
Table 1 Search strategy for MEDLINE (OvidSp)

\begin{tabular}{ll}
\hline 1 & Knee/or Knee Prosthesis/or Knee Joint/ \\
2 & Prosthesis-Related Infections/or Prosthesis Failure/or Periprosthetic Fractures/ \\
3 & (Revision knee replacement or revision knee arthroplasty). ti, ab, kw, tw \\
4 & Knee revision. ti, ab, kw, tw \\
5 & Reoperation. ti, ab, kw \\
6 & 1 or 2 or 3 or 4 or 5 \\
7 & Health Care Costs/or "Cost of Illness"/or Hospital Costs/ \\
8 & (Cost-Benefit Analysis or Cost-effectiveness Analysis or Cost-utility Analysis). ti, \\
9 & ab \\
10 & (Economic Burden or Costs or Cost Analysis). ti, ab, kw, tw \\
11 & 7 or 8 or 9 \\
12 & 6 and 10 \\
13 & Limit 11 to English language \\
\hline
\end{tabular}

$t i$ title, $a b$ abstract, $k w$ key word, $t w$ text word 
Table 2 Items on the article screening form

\begin{tabular}{ll}
\hline Phase 1, screening & Research article (yes/no) \\
& Research on revision total knee replacement (yes/no) \\
& Original research article (yes/no) \\
& Health economic evaluation (yes/no) \\
& Evaluation on revision knee replacement (yes/no) \\
& Evaluation involves cost (yes/no) \\
& Full-text/abstract presented in English language (yes/no) \\
& All phase I screening criteria met (yes/no) \\
Phase 2, eligibility (if all phase 1 & Costing method clear (yes/no) \\
screening criteria are met) & Cost perspective (societal, patient/payer/health provider) \\
& Components of the direct cost (intervention/laboratory test/service cost) \\
& Others (specify) \\
Components of indirect cost (productivity loss/lost productive years/ \\
transportation/unemployment) \\
Include article in the final data extraction (yes/no) \\
Any additional details about the article
\end{tabular}

from the two reviewers that cannot be resolved among themselves will be resolved by the third reviewer. The data will be selected based on the International Society for Pharmacoeconomics and Outcome Research (ISPOR) Consolidated Health Economic Evaluation Reporting Standards (CHEERS) guideline [19]. The Larg and Moss guideline for cost of illness will also be used in our data collection [20], and the Campbell and Cochrane Economics Methods Group (CCEGM) guideline will also be employed in our data extraction process [21].

\subsection{Data Items, Outcome and Prioritization}

Data will be extracted based on the following:

(a) Publication: Title, authors, year, and setting or country in which the study was conducted.

(b) Study design: Randomized controlled trials, cohort studies, case-control studies, etc.

(c) Aim of the study, sample size, sex, and comorbidities (see Table 3 for details).

(d) Cost measure: Our primary cost measure will be direct and indirect costs, such as productivity loss, cost of reduced work efficiency or workdays lost. Secondary outcomes include cost differences between primary TKR and revision TKR, number of revisions and incidence estimates.

The major cost drivers will be determined from the subcomponent costs extracted from each included study, as described in Table 3.
Table 3 Items on the data collection form for the included studies

\begin{tabular}{ll}
\hline 1 & Author \\
2 & Year of publication \\
3 & Year of study \\
4 & Research title and study objectives \\
5 & Cause of revision \\
7 & Comparator(s) \\
8 & Study setting \\
9 & Sample size \\
10 & Patient characteristics (age, sex, etc.) \\
11 & Study perspective \\
12 & Time horizon \\
13 & Discounting and price year, and currency \\
14 & Type of economic evaluation \\
15 & Study design \\
16 & Cost components (direct or indirect) \\
17 & Subcomponents of the cost (medications, laboratory, \\
& devices, surgical, productivity loss, transportation, service \\
& cost, etc.) \\
18 & Cost estimation approach: bottom-up or top-down \\
19 & Total cost \\
20 & Comorbidity \\
21 & Study assumptions \\
22 & Sensitivity analysis \\
23 & Conflict of interest \\
25 & Funding declaration \\
\hline & Any other relevant details \\
&
\end{tabular}




\subsection{Risk of Bias and Transparency in Individual Studies}

Assessment of the risk of bias and transparency in individual studies will be performed at the outcome level. The Consensus Health Economic Criteria (CHEC) checklist designed for conducting systematic reviews that are based on economic evaluation will be used to assess the risk of bias in the individual studies [22]. This checklist has 19 reporting standards for economic model characteristics, identification and valuation of costs and outcomes, discussion section, and conclusions, as well as funding and conflicts of interest. We will also use the Larg and Moss guideline to check for bias in the studies [20]. The risk-of-bias tools did not provide thresholds to include or exclude studies for data synthesis. We will use a conservative classification system to ensure that only studies with good costing approaches are included in our analysis. We will classify studies as 'low risk' (0-10\%), 'low-moderate risk' (11-20\%), 'high-moderate risk' (21-30\%), or 'high risk' (>30\%) based on applicable items for each study. Furthermore, we will evaluate the selected studies for transparency of cost estimates by using the criteria developed by Fukuda and Immanaka to classify the studies into transparency levels. The criteria consider the extent and clarity to which the cost components are reported, and the quantity and unit price of the resources reported in each study [23]. This transparency tool did not provide a threshold to include or exclude studies for data synthesis. Using its classification system, we will group studies into five levels and apply a conservative threshold to include studies for synthesis. The levels include 'excellent' $(\mathrm{A} \alpha)$, 'very good' (A $\beta, B \alpha, B \beta)$, 'good' $(B \gamma, C \alpha, C \beta, C \gamma)$, 'fair' $(\mathrm{B} \delta, \mathrm{C} \delta, \mathrm{D} \beta, \mathrm{D} \gamma)$ and 'poor' (D $\delta, \mathrm{D} \varepsilon$ ) transparency (see the Fukuda and Imanaka transparency tool for more details [23]). Studies with a high risk of bias (>30\%) and/or poor cost transparency ( $D \delta$ and $D \varepsilon$ ) will be excluded from the data synthesis. The checklists will be completed in duplicate by two members of the review team. Any differences will be resolved either with the third author or by review of other related referenced articles. Details of the risk-of-bias tools are shown in Online Resource 2 and Online Resource 3.

\subsection{Data Synthesis}

After extracting relevant data from the selected studies, a descriptive overview and characteristics of the studies will be presented in a tabular format. Studies with a high risk of bias and/or poor level of transparency will be excluded in the data synthesis but will be presented in a table. Furthermore, studies that presented cost as a health insurance reimbursement will be excluded because reimbursement costs do not represent the true costs of revision TKR and are often lower than the actual cost, but can sometimes be higher. Data synthesis and analysis will be performed in two phases. First, we will perform a narrative synthesis and summary of answers to our research questions. The result will be presented in a tabular format and a narrative summary. Descriptive statistical analysis may also be performed based on the information extracted.

Second, we will perform a quantitative synthesis of homogenous data if possible. Due to the expected heterogeneity in the cost estimates of the studies, only studies including the 'direct medical cost' will be synthesized quantitatively based on the studies' perspectives. There are complexities and variations in reporting indirect costs, therefore studies that present indirect costs will be synthesized narratively (first phase of the data synthesis). In a case where a study reports both direct medical cost and indirect cost, the direct medical cost component will be included in the 'direct medical cost' group for synthesis. Furthermore, there are variations in the characteristics of aseptic and septic revision TKR. Thus, the 'direct medical cost' group will be further grouped into aseptic and septic subgroups and synthesized separately. Grouping of studies for quantitative synthesis will also depend on the income level of each country whose data were reported (low, middle or high income). We expect the cost year and currency to vary across studies. Using a web-based tool developed by the CCEMG and the Evidence for Policy and Practice Information and Coordinating Centre (EPPI-Centre), we will estimate all costs to 2019 US dollars [24].

To quantify the effect of heterogeneity in each subgroup, a measure of the degree of inconsistency $\left(\mathrm{I}^{2}\right)$, which represents the extent to which the studies are inconsistent, will be undertaken. This describes the percentage of total variation across studies that are due to heterogeneity rather than chance. The $\mathrm{I}^{2}$ is calculated as $\mathrm{I}^{2}=100 \% \times(\mathrm{Q}-\mathrm{df}) / \mathrm{Q}$, where $Q$ is Cochran's heterogeneity statistic and $\mathrm{df}$ is the degrees of freedom. Negative values of $\mathrm{I}^{2}$ will be represented as zero so that $\mathrm{I}^{2}$ lies between $0 \%$ and $100 \%$. A value of $0 \%$ indicates no observed heterogeneity, while values above $50 \%$ will imply substantial heterogeneity [25]. A meta-analysis of studies in each subgroup in the 'direct medical cost' group will be performed to obtain the pooled cost estimate from the individual studies with similar characteristics based on the country's income level and type of revision (aseptic or septic revision TKR). A forest plot will be used to visualize the extent of heterogeneity among studies. Since heterogeneity is expected in the cost estimates between the studies, we will adopt the random-effect model. The forest plot will show the individual study cost estimate with Clopper-Pearson confidence intervals (CIs) and the overall (combined) DerSimonian-Laird pooled estimate.

We will also perform a meta-bias assessment across the studies included in the meta-analysis, for each subgroup, to assess the possibility of outcome bias due to selective 
reporting within the studies. Visual assessment will be undertaken using funnel plots of the mean cost to the standard error for each meta-analysis subgroup. A formal analysis will also be performed using Egger's test. In cases where the Egger's test shows a $p$ value $<0.05$, this implies a high heterogeneity level, therefore the meta-analysis of the subgroup(s) will not be reported. The analysis will be performed using StatsDirect (version 3) statistical software.

\section{Discussion}

This protocol provides a proposed stepwise plan for conducting a systematic review and meta-analysis of the cost of revision TKR. The research questions that will be answered in the Results section will be discussed in the Discussion section. The review will provide a narrative summary and a pooled estimate of the cost (for homogenous studies). It will also provide information about the studies' characteristics and identify major cost drivers of revision TKR, along with possible recommendations to minimize the cost. Furthermore, this study may inform the decisions of future revision TKR procedures in terms of cost minimization.

\section{Acknowledgements Not applicable.}

\section{Declarations}

Ethical approval and consent to participate Not applicable.

Consent for publication Not applicable.

Availability of data and materials Data sharing is not applicable in this article as no datasets were generated or analysed during the current study.

Competing interest Charles Okafor, Son Nghiem, Christopher Vertullo and Joshua Byrnes declare they have no competing interests.

Funding The authors received no funding for this research.

Author contributions $\mathrm{CO}, \mathrm{JB}$ and $\mathrm{CV}$ were responsible for conceptualization. $\mathrm{CO}$ and $\mathrm{SN}$ developed the study design, and the inclusion and exclusion criteria. $\mathrm{CO}, \mathrm{CV}, \mathrm{JB}$ and $\mathrm{SN}$ contributed to the synthesis and data analyses methods. $\mathrm{CO}$ wrote the first draft of the protocol, and $\mathrm{JB}, \mathrm{SN}$ and $\mathrm{CV}$ made the corrections. All authors reviewed the final manuscript submitted for publication.

Open Access This article is licensed under a Creative Commons Attribution-NonCommercial 4.0 International License, which permits any non-commercial use, sharing, adaptation, distribution and reproduction in any medium or format, as long as you give appropriate credit to the original author(s) and the source, provide a link to the Creative Commons licence, and indicate if changes were made. The images or other third party material in this article are included in the article's Creative Commons licence, unless indicated otherwise in a credit line to the material. If material is not included in the article's Creative Commons licence and your intended use is not permitted by statutory regulation or exceeds the permitted use, you will need to obtain permission directly from the copyright holder. To view a copy of this licence, visit http://creativecommons.org/licenses/by-nc/4.0/.

\section{References}

1. American Academy of Orthopaedic Surgeons. Revision Total Knee Replacement. 2015. https://orthoinfo.aaos.org/en/treatment/ revision-total-knee-replacement/. Accessed 15 April 2020.

2. Australian Orthopaedic Association. Joint Replacement Annual Report: 20th Annual Report. 2019. https://aoanjrr.sahmri.com/ annual-reports-2019. Accessed 15 April 2020.

3. Le DH, Goodman SB, Maloney WJ, Huddleston JI. Current modes of failure in TKA: infection, instability, and stiffness predominate. Clin Orthop Relat Res. 2014;472(7):2197-200.

4. Brittain R, Young E, Mccormack V, Swanson M. 16th Annual Report 2019: National Joint Registry for England, Wales, Northern Ireland and the Isle of Man. 2019 (December 2018). https:// www.hqip.org.uk/wp-content/uploads/2018/11/NJR-15th-Annua 1-Report-2018.pdf. Accessed 19 April 2020.

5. Lund University. Swedish Knee Arthroplasty Register: 2019 Annual Report. Lund; 2019. http://myknee.se/en/. Accessed 19 April 2020.

6. Haukeland University. Norwegian Arthroplasty Register Report 2019. Vol. 8906. 2019. Available at: http://nrlweb.ihelse.net/eng/. Accessed 11 April 2020.

7. Kulshrestha V, Datta B, Mittal G, Kumar S. Epidemiology of revision total knee arthroplasty: a single center's experience. Indian J Orthop. 2019;53(2):282-8.

8. Kurtz SM, Ong KL, Lau E, Widmer M, Maravic M, Gómez-Barrena $\mathrm{E}$, et al. International survey of primary and revision total knee replacement. Int Orthop. 2011;35(12):1783-9.

9. Oduwole KO, Molony DC, Walls RJ, Bashir SP, Mulhall KJ. Increasing financial burden of revision total knee arthroplasty. Knee Surg Sport Traumatol Arthrosc. 2010;18(7):945-8.

10. Ritter MA, Eizember LE, Fechtman RW, Keating EM, Faris PM Revision total knee arthroplasty. A survival analysis. J Arthroplasty. 1991;6(4):351-6.

11. Saleh KJ, Rand JA, McQueen DA. Current status of revision total knee arthroplasty: how do we assess results? JBJS. 2003;85(Suppl 1):S18-20.

12. Greidanus NV, Peterson RC, Masri BA, Garbuz DS. Quality of life outcomes in revision versus primary total knee arthroplasty. J Arthroplasty. 2011;26(4):615-20.

13. Hebert CK, Williams RE, Levy RS, Barrack RL. Cost of treating an infected total knee replacement. Clin Orthop Relat Res. 1996:331:140-5.

14. Kallala RF, Ibrahim MS, Sarmah S, Haddad FS, Vanhegan IS Financial analysis of revision knee surgery based on NHS tariffs and hospital costs. Does it pay to provide a revision service? Bone Jt J. 2015;97-B(2):197-201.

15. Vertullo CJ, Graves SE, Peng Y, Lewis PL. An optimum prosthesis combination of low-risk total knee arthroplasty options in all five primary categories of design results in a $60 \%$ reduction in revision risk: a registry analysis of 482,373 prostheses. Knee Surg Sport Traumatol Arthrosc. 2019;27(5):1418-26.

16. Moher D, Shamseer L, Clarke M, Ghersi D, Liberati A, Petticrew $\mathrm{M}$, et al. Preferred reporting items for systematic review and meta-analysis protocols (PRISMA-P) 2015 statement. Syst Rev. 2015;(January):1-9.

17. Bramer WM, Rethlefsen ML, Kleijnen J, Franco OH. Optimal database combinations for literature searches in systematic reviews: a prospective exploratory study. Syst Rev. 2017;6(1):1-12. 
18. AcademyHealth. Health Economics Core Library Recommendations, 2011. https://www.nlm.nih.gov/nichsr/corelib/hecon-2011. html. Accessed 20 April 2020.

19. Husereau D, Drummond M, Petrou S, Carswell C, Moher D, Greenberg D, et al. Consolidated Health Economic Evaluation Reporting Standards (CHEERS) - explanation and Elaboration: a Report of the ISPOR Health Economic Evaluation Publication Guidelines Good Reporting Practices Task Force. Value Health. 2013;16(2):231-50.

20. Larg A, Moss JR. Cost-of-illness studies. Pharmacoeconomics. 2011;29(8):653-71.

21. Shemilt I, Mugford M, Byford S, Drummond M, Eisenstein E, Knapp M, et al. Incorporating economics evidence. In: Higgins J, Green S (eds). Cochrane handbook for systematic reviews of interventions. Chapter 15. Oxford: The Cochrane Collaboration; 2011. http://www.cochrane-handbook.org. Accessed 14 April 2020.
22. Evers S, Goossens M, de Vet H, van Tulder M, Ament A. Criteria list for assessment of methodological quality of economic evaluations: consensus on Health Economic Criteria. Int J Technol Assess Health Care. 2005;21(2):240-5.

23. Fukuda H, Imanaka Y. Assessment of transparency of cost estimates in economic evaluations of patient safety programmes. $\mathbf{J}$ Eval Clin Pract. 2009;15(3):451-9.

24. CCEMG and EPPI-Centre. CCEMG-EPPI-Centre Cost Converter v.1.6. 2019. https://eppi.ioe.ac.uk/costconversion/defau lt.aspx. Accessed 15 March 2020.

25. Higgins JPT, Thompson SG. Quantifying heterogeneity in a metaanalysis. Stat Med. 2002;21(11):1539-58. 